\title{
Complete clinical response of a patient with lymph node and pulmonary metastatic hepatocellular carcinoma to very low-dose sorafenib $\left(\right.$ Nexavar $\left.^{\circledR}\right)$ therapy
}

\author{
Yutaka Takahashi $\cdot$ Satoshi Katagiri $\cdot$ \\ Shun-ichi Ariizumi - Yoshihito Kotera • \\ Hiroto Egawa $\cdot$ Masakazu Yamamoto
}

Received: 18 October 2011/ Accepted: 27 June 2012

(C) The Japan Society of Clinical Oncology 2012

\begin{abstract}
We present the case of a patient with metastatic hepatocellular carcinoma (HCC) who showed complete response (CR) to very low-dose sorafenib therapy. The patient was a 74-year-old woman who had previously undergone S7/8 hepatectomy for HCC in December 2002. Intrahepatic recurrence developed in August 2007, and transcatheter arterial chemoembolization was performed seven times. An increase in the level of the tumor marker alpha-fetoprotein (AFP) was observed, and thus positron emission tomography-computed tomography (PET-CT) was performed in June 2010. PET-CT showed an accumulation of metastatic cells resulted in a swollen periaortic lymph node. Based on the features of this lymph node metastasis, HCC was diagnosed. Oral sorafenib (Nexa$\operatorname{var}^{\circledR}$ ) was started at an initial dose of $400 \mathrm{mg} /$ day given three times a week. Sorafenib therapy was discontinued when adverse reactions such as bloody sputum and hand tremor were noted after 1 week of therapy. Following improvement of the adverse reactions, sorafenib therapy was resumed at $200 \mathrm{mg} /$ day given three times a week. Two months after the reinitiation of sorafenib therapy, CT showed no change in the lymph node metastasis, and previously undetected pulmonary metastasis was diagnosed. At 5 months of therapy, CT showed no pulmonary and lymph node metastases. As the patient developed another adverse effect of sorafenib therapy, the frequency of sorafenib administration was reduced to two times a week at $200 \mathrm{mg}$ /day. After 15 months of sorafenib therapy at
\end{abstract}

Y. Takahashi $(\bowtie) \cdot$ S. Katagiri · S. Ariizumi · Y. Kotera ·

H. Egawa $\cdot$ M. Yamamoto

Department of Surgery, Institute of Gastroenterology,

Tokyo Women's Medical University, 8-1 Kawada-cho,

Shinjuku-ku, Tokyo 162-8666, Japan

e-mail: yutaka@ige.twmu.ac.jp
$200 \mathrm{mg} /$ day given two times a week, the patient showed $\mathrm{CR}$ and remains in $\mathrm{CR}$ at this writing.

Keywords Complete clinical response - Hepatocellular carcinoma $\cdot$ Sorafenib

\section{Introduction}

Recent advances in molecular biology have elucidated the mechanisms of cancer growth, invasion and metastasis. At present, molecular target drugs are being used as the standard therapy for different types of tumor. In Japan, the indication of sorafenib (Nexavar ${ }^{\circledR}$ ) for hepatocellular carcinoma (HCC) that is not treatable by curative resection was approved in May 2009, and sorafenib is currently being used considerably in medical practice. Sorafenib has been demonstrated to prolong the overall survival of HCC patients in large-scale clinical studies conducted to validate its approval as standard therapy $[1,2]$. In these previous studies, no complete clinical responders were identified. However, complete response (CR) cases have been reported occasionally, which has attracted increasing interest regarding the efficacy of sorafenib against HCC.

\section{Case report}

The patient was a 74-year-old woman who underwent complete examination for liver disorders in April 1988 and was given a diagnosis of chronic hepatitis B. In December 2002, the patient underwent S7/8 hepatectomy for HCC (35-mm moderately differentiated HCC vp1, vv0, b0, im2; stage III, The Liver Cancer Study Group of Japan). Immunotherapy was given as postoperative adjuvant 
Fig. 1 Clinical course of the patient. $A F P$ alpha-fetoprotein, $P I V K A-2$ protein induced by vitamin $\mathrm{K}$ abnormality, TACE transcatheter arterial chemoembolization

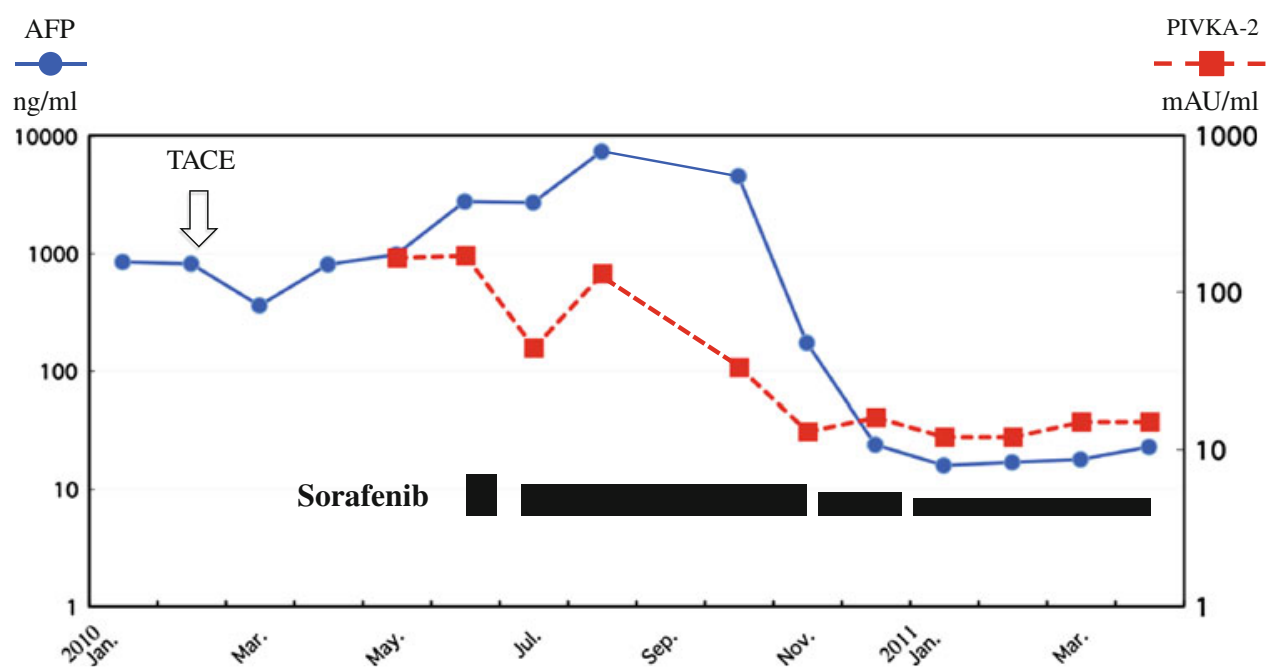

PIVKA-2

$\mathrm{mAU} / \mathrm{ml}$

000

100

(CTCAE) version 3.0: grade 1] and tremor of the fingers (CTCAE version 3.0: grade 2) were noted, and thus the therapy was discontinued. Following improvement of the adverse reactions, sorafenib administration was restarted at a reduced dose of $200 \mathrm{mg} /$ day given three times a week. After 2 months of sorafenib therapy, CT showed no change in lymph node metastasis, but a new $\varphi$ 8-mm pulmonary metastasis developed in the right upper lung field (Fig. 4a). The AFP level started to decrease at 4 months of sorafenib therapy, and both pulmonary and lymph node metastases disappeared on CT at 5 months of sorafenib therapy (Figs. 3b, 4b). Because facial flush as an adverse reaction (CTCAE version 3.0: grade 2) developed, the dose of sorafenib was further reduced to $200 \mathrm{mg}$ /day given two times a week, and treatment was continued for several months. At 7 months of sorafenib therapy, PET-CT showed no periaortic lymph node enlargement or abnormal contrast accumulation (Fig. 2c, d). As of 15 months of sorafenib therapy at $200 \mathrm{mg} /$ day given two times a week, the patient has continued to be in $\mathrm{CR}$ without tumor recurrence on imaging examinations. Moreover, the AFP level has remained low.

\section{Discussion}

The tumor growth-inhibitory effects of sorafenib have been demonstrated to prolong survival in large-scale clinical studies, namely, the Sorafenib HCC Assessment Randomized Protocol (SHARP) trial and Asia-Pacific studies, both conducted before the approval of the indication of sorafenib for HCC in Japan [1, 2]. Although the survival-prolongation effect of sorafenib has been verified in these previous studies, its tumor size-reducing effect is not very pronounced. Also, no $\mathrm{CR}$ cases were noted in these previous large-scale clinical studies. However, several CR cases have recently been reported. In Japan, 15 CR cases were reported from multiple centers, as stated in the report of the Japan Association of 
Fig. 2 18F-

fluorodeoxyglucose-positron emission tomography-computed tomography. a, b Before treatment with sorafenib. $\mathbf{c}, \mathbf{d}$ At 7 months of sorafenib administration
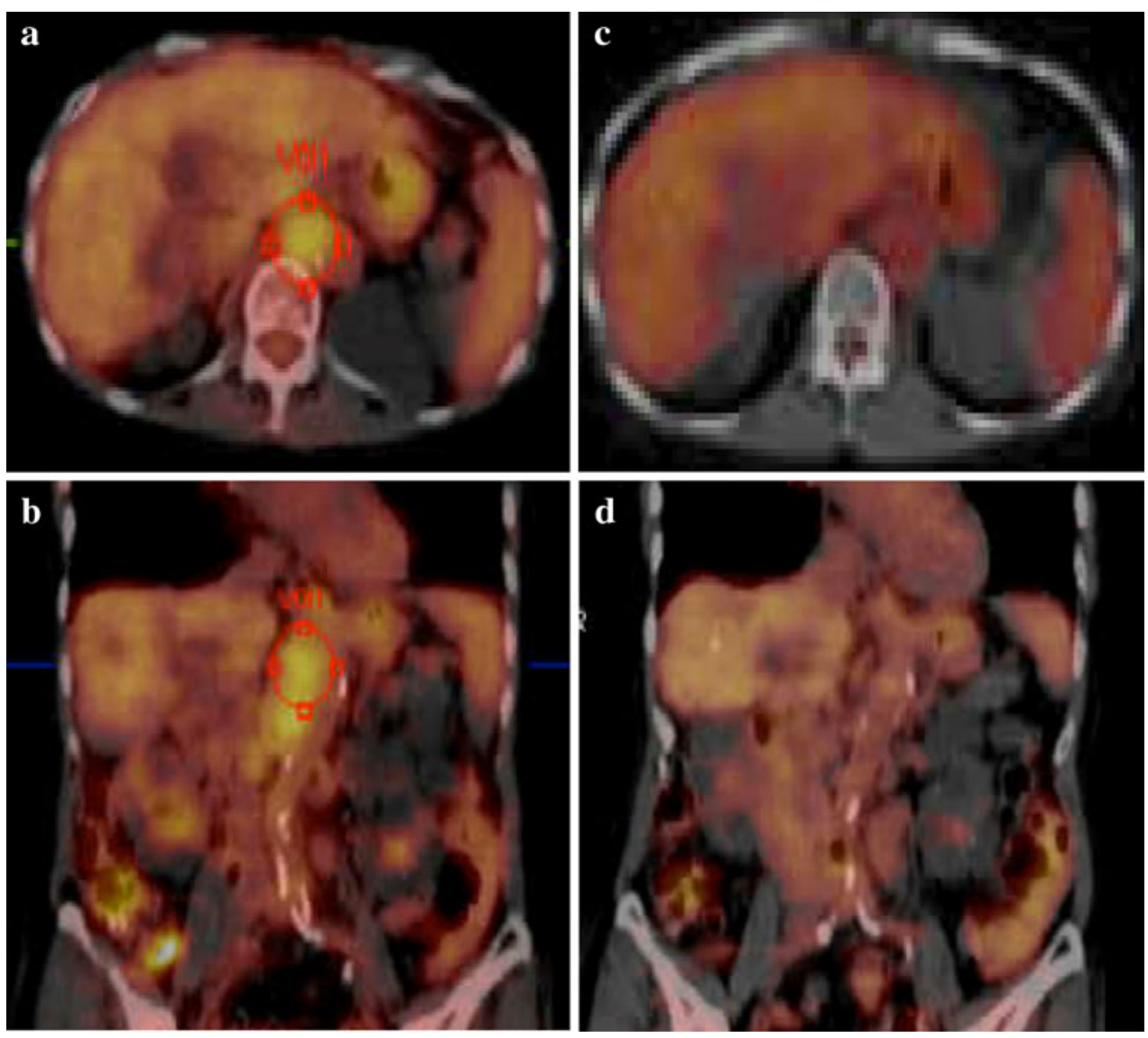

Fig. 3 Abdominal enhanced computed tomography. a Before treatment with sorafenib. b At 5 months of sorafenib administration
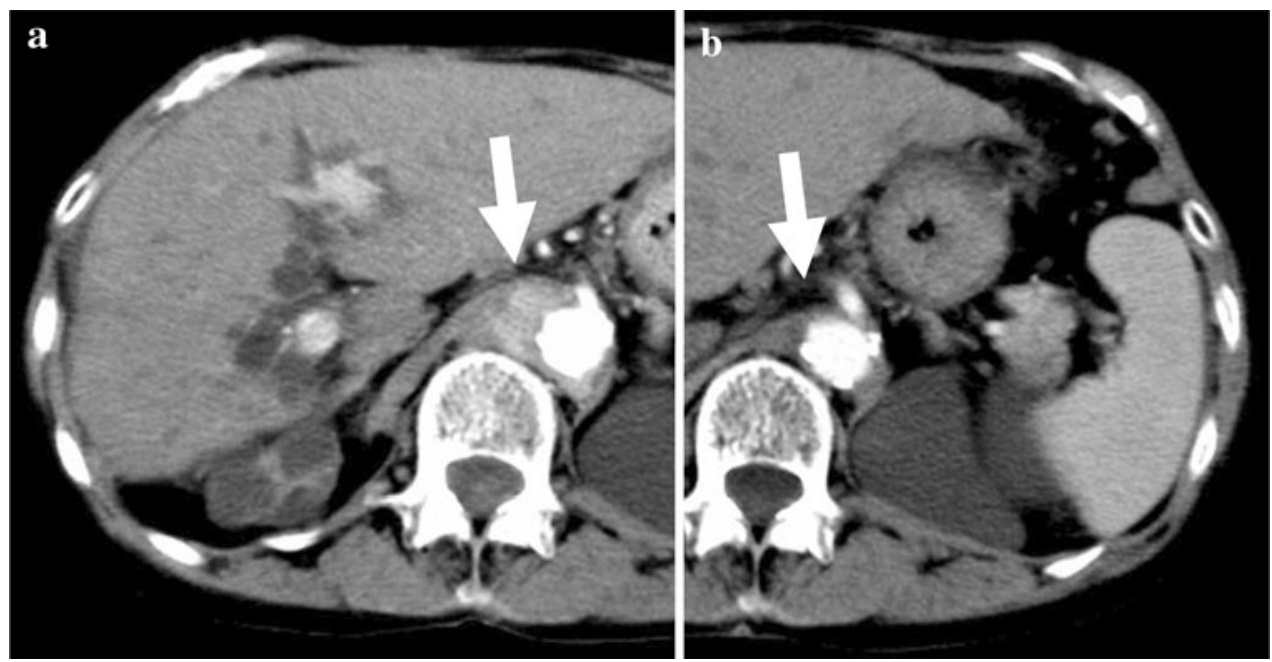

Molecular Targeted Therapy [3]. This suggests that some Japanese HCC cases are highly sensitive to sorafenib.

In the present patient, the absence of intrahepatic lesions has been maintained for more than 4 months after the disappearance of all tumors, including the lymph node and pulmonary metastases. The patient is thus considered to be in CR, as indicated by the absence of intense staining, shrinking, and disappearance of the tumors.
For the evaluation of the effects of anticancer drugs on solid cancers, the Response Evaluation Criteria in Solid Tumors (RECIST) are widely used. RECIST indicates the tumor size-reducing effect of a given therapy, but are considered by others as not applicable for the evaluation of a given therapy for HCC. For example, the sorafenibinduced disappearance of intense tumor staining is not considered to indicate effective therapy. In Japan, the effect 
of TACE is comprehensively assessed using the Response Evaluation Criteria in Cancer of the Liver (RECICL) [4] based on the disappearance of intense staining and size reduction rate of a tumor. In Western countries, the modified RECIST (mRECIST) for HCC, which consider regions showing the disappearance of intense tumor staining as indicative of tumor necrosis, have been proposed [5] and used for evaluating treatment effect on HCC. On the other hand, some researchers doubt the merit of evaluating treatment effect based on the disappearance of intense tumor staining. Schor-Bardach et al. [6] reported that tumor size was not changed despite the sorafenib-induced reduction of blood flow in the tumor region in a mouse human renal cell carcinoma-transplanted model. Moreover, blood flow in the tumor region and tumor size increased despite the continuation of sorafenib therapy. This suggests that sorafenib-induced

Table 1 Laboratory data before sorafenib treatment

\begin{tabular}{ll}
\hline WBC $4,010 / \mathrm{mm}^{3}$ & Alb $3.3 \mathrm{~g} / \mathrm{dl}$ \\
RBC $296 \times 10^{4} / \mathrm{mm}^{3}$ & AST 33 IU/l \\
$\mathrm{Hb} 8.6 \mathrm{~g} / \mathrm{dl}$ & ALT 16 IU/l \\
Plt $22.7 \times 10^{4} / \mathrm{mm}^{3}$ & ALP $436 \mathrm{IU} / \mathrm{l}$ \\
PT $89.1 \%$ & T-bil $0.5 \mathrm{mg} / \mathrm{dl}$ \\
PT-INR 1.00 & BUN $33.2 \mathrm{mg} / \mathrm{dl}$ \\
AFP 2,786 ng/ml & Cr $1.64 \mathrm{mg} / \mathrm{dl}$ \\
AFP-L3 $1.3 \%$ & CRP $0.58 \mathrm{mg} / \mathrm{dl}$ \\
PIVKA-2 $171 \mathrm{mAU} / \mathrm{ml}$ & HBsAg $(+)>2,000$ \\
& HB DNA real-time $<2.1 \mathrm{log} \mathrm{IU} / \mathrm{ml}$ \\
& HCVAb $(-)$ \\
\hline
\end{tabular}

reduction of blood flow in the tumor region does not immediately lead to tumor size reduction or necrosis. Specifically, the primary mechanisms of action of sorafenib are the induction of necrosis by inhibition of vascularization and the induction of apoptosis [7]. This leaves a question of whether the evaluation criteria for the therapeutic efficacy of sorafenib are simply based on the induction and development of tumor necrosis. Further investigation to clarify the basis of therapeutic efficacy using these criteria is necessary.

A high frequency of adverse reactions, such as hand-foot syndrome, and relatively high frequencies of skin eruption and diarrhea are also characteristics of sorafenib therapy. In our experience at our hospital, sorafenib was initially given to more than 50 patients with unresectable $\mathrm{HCC}$ at the standard dose of $800 \mathrm{mg} /$ day; however, this initial dose was reduced to a one-step lower dose at $400 \mathrm{mg} /$ day because of the high frequency of adverse reactions. It has been reported that when the rates of adverse events were compared between phase I clinical studies performed in Japan and other countries, there were no marked differences in the type, symptoms, findings, severity, or incidence between Japanese and non-Japanese patients. Based on this finding, the main trend in sorafenib therapy is to set the initial dose as close as possible to $800 \mathrm{mg} /$ day. However, the use of a lower dose of sorafenib at $400 \mathrm{mg} / \mathrm{day}$ has been reported in Japan in cases of increased frequency of treatment or continuous therapy because of the use of an initial reduced dose [8]. This does not mean that Japanese patients offer an effective treatment group for sorafenib. Further studies regarding the optimal dose of sorafenib for HCC are needed.
Fig. 4 Chest enhanced computed tomography. a A new $\varphi 8$-mm pulmonary metastasis developed in the right upper lung field after 2 months. b Pulmonary metastasis disappeared at 5 months
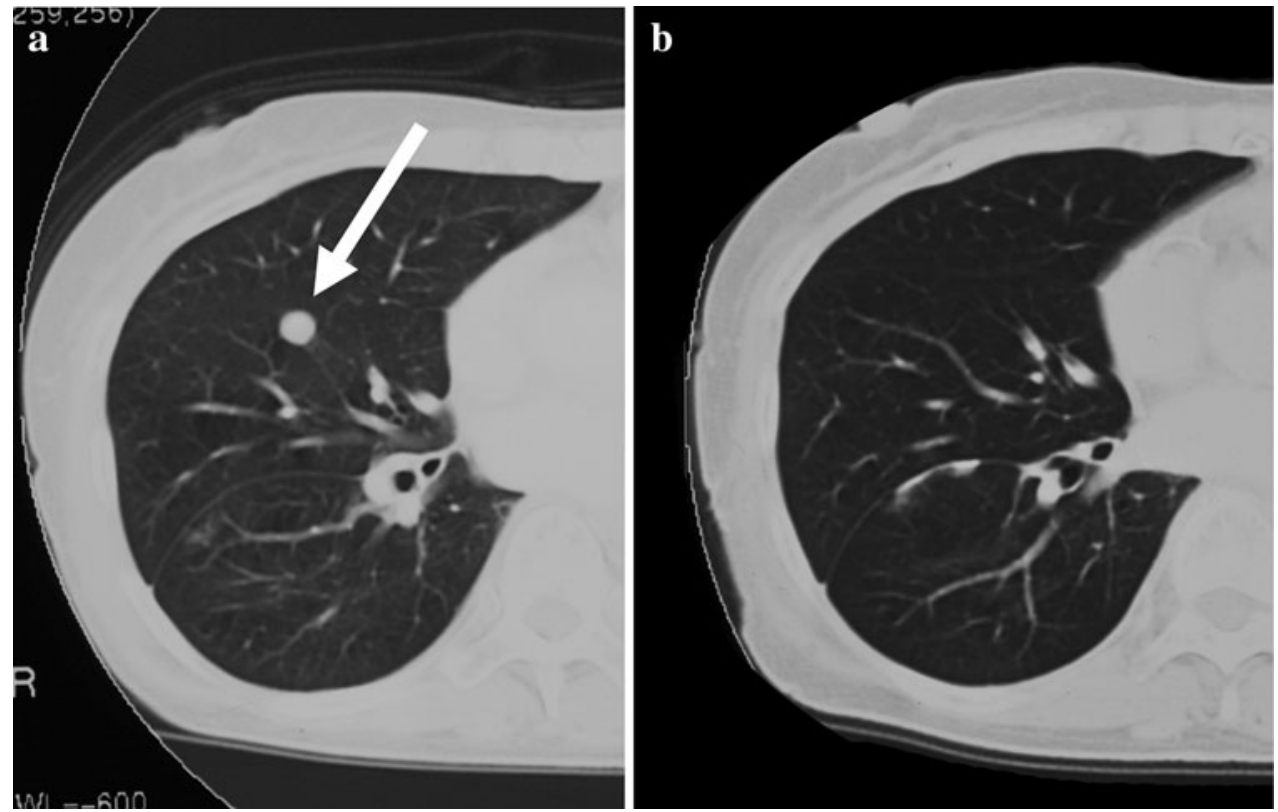
Table 2 Recent reports describing CR cases
${ }^{a}$ Dose administered (mg/day)

b Tumor location of HCC (and the site of metastasis)

c Published year/country/ reference number

\begin{tabular}{llllll}
\hline Case & Sex & Age & Dose $^{\text {a }}$ & Location $^{\text {b }}$ & Published $^{\text {c }}$ \\
\hline 1 & M & 74 & 400 & Liver & 2010/USA/[10] \\
2 & M & 68 & 400 & Liver, lung & 2010/Japan/[9] \\
3 & M & 68 & 800 & Liver, lung, lymph node, adrenal & \\
4 & M & 69 & 800 & Liver, lymph node & 2010/Greece/[11] \\
5 & M & 84 & 800 & Liver (PVTT) & 2011/Italy/[12] \\
6 & M & 56 & 800 & Liver & 2011/France/[13] \\
7 & M & 59 & 800 & Liver (PVTT), lymph node, dissemination, & 2011/France/[14] \\
8 & M & 76 & 400 & Liver, lung & 2011/Japan/[15] \\
\hline
\end{tabular}

Sorafenib requires a specific period to exhibit its dosedependent effect, although its effect is directly related to the dose. Kudo et al. [9] reported that 2 of 90 patients treated with sorafenib achieved CR. In these two patients, the tumor disappeared after 2 months of oral sorafenib administration at $400 \mathrm{mg} /$ day in one patient, whereas an intrahepatic tumor and pulmonary and lymph node metastases disappeared after 1 month and an adrenal metastatic lesion shrank after 6 months in the other patient treated with sorafenib at 800 $\mathrm{mg} /$ day. Complete necrosis was pathologically confirmed in the excised specimens. We searched recent reports describing CR cases [9, 10] (Table 2). However, there were no reports of effective cases of very low-dose sorafenib therapy.

So et al. [16] reported that intrahepatic lesions disappeared after 5 months of oral sorafenib administration at $400 \mathrm{mg} /$ day. Lassau et al. [17, 18] reported marked tumor size reduction after 6 months in HCC patients treated with the molecular target drug bevacizumab; in these patients reduced tumor blood flow early after treatment initiation was confirmed by contrast echography. In the present patient, sorafenib administration was initiated at about $21 \%$ of the standard dose per week (i.e., $400 \mathrm{mg}$ /day given 3 times a week), and the dose was further reduced twice because of adverse reactions. The dose has been maintained at about $7 \%$ of the standard dose $(200 \mathrm{mg} /$ day, given 2 times a week), and CR was achieved after 5 months and maintained for more than 4 months. It is suggested that sorafenib administration must be continued when its dose is reduced in individual cases as CR may be achieved by continuous lowdose therapy. Taken together, this is an important case that showed that long-term low-dose sorafenib therapy achieved $\mathrm{CR}$ in terms of lymph node and pulmonary metastases in a patient following surgery for HCC.

Conflict of interest No author has any conflict of interest.

\section{References}

1. Llovet JM, Ricci S, Mazzaferro V et al (2008) Sorafenib in advanced hepatocellular carcinoma. N Engl J Med 359:378-390
2. Cheng AL, Kang YK, Chen Z et al (2009) Efficacy and safety of sorafenib in patients in the Asia-Pacific region with advanced hepatocellular carcinoma a phase III randomised, double-blind, placebo-controlled trial. Lancet Oncol 10:25-34

3. Japan Association of Molecular Targeted Therapy (2010) 1st meeting abstract book Osaka, Japan Association of Molecular Targeted Therapy

4. Kudo M, Kubo S, Takayasu K et al (2010) Response evaluation criteria in cancer of the liver (RECICL). Kanzo 51:261-266

5. Lencioni R, Llovet JM (2010) Modified RECIST (mRECIST) assessment for hepatocellular carcinoma. Semin Liver Dis 30: $52-60$

6. Schor-Bardach R, Alsop DC, Pedrosa I et al (2009) Does arterial spin-labeling MR imaging-measured tumor perfusion correlate with renal cell cancer response to antiangiogenic therapy in a mouse model? Radiology 251:731-742

7. Wilhelm SM, Carter C, Tang L et al (2004) BAY 43-9006 exhibits broad spectrum oral antitumor activity and targets the RAF/MEK/ ERK pathway and receptor tyrosine kinases involved in tumor progression and angiogenesis. Cancer Res 64:7099-7109

8. Numata K, Terada M, Morimoto M et al (2010) Comparison between adverse events of half-dose and standard dose administration of sorafenib for advanced hepatocellular carcinoma. Kanzo 51:400-402

9. Kudo M, Ueshima K (2010) Positioning of a molecular-targeted agent, sorafenib, in the treatment algorithm for hepatocellular carcinoma and implication of many complete remission cases in Japan. Oncology 78(suppl 1):154-166

10. Wang SX, Byrnes A, Verma S et al (2010) Complete remission of unresectable hepatocellular carcinoma treated with reduced dose of sorafenib: case report. Target Oncol 5:59-63

11. Chelis L, Ntinos N, Souftas V et al (2011) Complete response after sorafenib therapy for hepatocellular carcinoma in an HIVHBV co infected patient: possible synergy with HAART? A case report. Med Oncol 28:S165-S168

12. Sacco R, Bargellini I, Gianluigi G et al (2011) Complete response for advanced liver cancer during sorafenib therapy: case report. BMC Gastroenterol 11:4

13. Curtit E, Thiery-Vuillemin A, Nguyen T et al (2011) Complete histologic response induced by sorafenib in advanced hepatocellular carcinoma: a case report. J Clin Oncol 29:330-332

14. Irtan S, Chopin-Laly X, Ronot M et al (2011) Complete regression induced by sorafenib of locally advanced hepatocellular carcinoma allowing curative resection. Liver Int 31:740-743

15. Inuzuka T, Nishikawa H, Sekikawa A et al (2011) Complete response of advanced hepatocellular carcinoma with multiple lung metastases treated with sorafenib: a case report. Oncology 81(suppl 1):152-157

16. So BJ, Bekaii-Saab T, Bloomston MA et al (2008) Complete clinical response of metastatic hepatocellular carcinoma to 
sorafenib in a patient with hemochromatosis: a case report. J Hematol Oncol 1:18

17. Lassau N, Chami L, Benatsou B et al (2007) Dynamic contrastenhanced ultrasonography (DCE-US) with quantification of tumor perfusion: a new diagnostic tool to evaluate the early effects of antiangiogenic treatment. Eur Radiol Suppl 17(Suppl 6):89-98

18. Lassau N, Lamuraglia M, Chami L et al (2006) Gastrointestinal stromal tumors treated with imatinib: monitoring response with contrast-enhanced sonography. AJR Am J Roentgenol 187:1267-1273 